\title{
PRE-WHITE DWARF EVOLUTION: UP TO PLANETARY NEBULAE
}

\author{
Italo Mazzitelli \\ Istituto di Astrofisica Spaziale del CNR \\ Via E. Fermi 21, 00044 Frascati, Italy
}

\begin{abstract}
The main evolutionary phases having some interest for the formation of the remnant white dwarf are discussed, starting from the core helium burning phase, in the attempt of evaluating a theoretical relation between initial main sequence mass and final white dwarf mass. Several difficulties, mainly due (but not only) to uncertainties in the theory of mass loss, have been met, so that only a fiducial bona fide correlation can be drawn. The mass function of population I white dwarfs has probably a secondary maximum at $M=0.9-1 \mathrm{Me}_{\theta}$.
\end{abstract}

\section{INTRODUCTION}

In a review of this kind it is very difficult to correctly quote all the researchers who, in the last thirty years, have contributed to our present understanding of the pre-white dwarf stellar evolution. In the following, several results will be given for granted, without explicit quotations, apart from a few cases; it is however to be noted that the most of the discussion will be based upon works by Schönberner, Iben, Becker, Lattanzio, the Padua group (Bertelli, Bressan, Chiosi and coworkers), Packzynski, Wood, Faulkner, D'Antona and myself and, in general, upon the results by people who afforded the risk to plug their hands in the mess of numerical computations. Our thanks to those people who have the courage to do the dirty job. which can be always easily criticized, but which is at the very ground of most improvements in astrophysical knowledge, at the same level as the observational activity.

In principle, the stellar evolution through the wD state could be studied without a previous understanding of the preceeding evolutionary phases (D'Antona 1988). A parametrical approach in which total mass and chemical distribution in the interior can be arbitrarily chosen, is however almost meaningless when the comparison 
to the observational data is tried (Mazzitelli and D'Antona 1987), since the initial choice of parameters severely affects the following evolution. For this reason, in the last ten years a number of attempts have been performed to follow the whole stellar evolution from the main sequence to the white dwarf stage (Schönberner 1979, 1981, 1983, 1987a Iben et al: 1983, Iben 1984, Iben and Tutukov 1984, Mazzitelli and D'Antona 1986a Wood and Faulkner 1986), to determine the proper, physically correct choice for the initial white dwarf parameters. In the following. I will try to summarize our present level of understanding of the evolutionary processes having some influence upon the star when it reaches its white dwarf stage. I will limit the discussion to Population I stars, since at present the vast majority of observational informations upon white dwarfs is relative to the solar environment. Since in any case I am left with a mammoth task, I will further restrict the subject, trying to focus my attention upon only one of the various aspects relevant for the understanding of the white dwarfs properties, that is: the relation between initial main sequence mass $\left(M_{1 n}\right)$ and the final white dwarf mass ( $\left.M_{1}{ }_{1 n}\right)$. Of course, a number of questions cannot yet be solved by theory alone, and I will be forced, in some cases, to take advantage from the observations in the tuning of some free parameters, especially for what concerns the mass loss and/or envelope ejection mechanisms; nevertheless I will always try to stay on the theoretical, modelistic side of the subject, as my intention is to give clues about the theoretical relatively safe conclusions which can be raised, and on those which are still subject to debate, for which there is still room for substantial revisions.

\section{STARS EXPERIENCING THE CENTRAL HELIUM FLASH}

Let me start by fixing boundaries and internal subdivisions to the range of initial masses which can give rise to white dwarfs.It is very hard to expect disk white dwarfs coming from Population I stars having mass smaller than the solar one, since the pre-white dwarf evolutionary life of the sun is just of the order of the galactic age. on the other side, there is still no agreement about the maximum initial mass which can die as a white dwarf. In fact, most of the recent computations (Castellani et Al., 1985) seem to show that a star of initial mass about 7 Me ignites carbon off-center in a degenerate core leading, perhaps, to the distruction of the star. Detailed hydrodynamic computations of this carbon ignition are still far from our reach (Iben 1982) but, as we will see, the possibility of a 
quiescent carbon burning with the formation of neon-magnesium white dwarfs of mass larger than 1.05-1.1 $\mathrm{M}_{\theta}$ cannot be excluded, at least on the observational ground, so that the upper limiting mass for the formation of white dwarfs can be shifted up to about $9 \mathrm{M}_{0}$ (Iben 1987). In the following I will limit my discussion to stars which do not reach the carbon ignition, but this last possibility has to be taken seriously into account.

The range 1 to $7 \mathrm{Me}$ can be further divided into three subranges, namely:

i) stars undergoing the central helium flash $\left(M<2.4 \mathrm{M}_{\theta}\right)$;

ii) stars gently igniting Helium, and not undergoing the second dredge-up ( $M=2.4 \div 4.5 \mathrm{Me}$, Becker and Iben 1979);

iii) stars undergoing the second dredge-up $(M>4.5 \mathrm{Me})$.

Let me start from the first mass range. There seems to be at present a general agreement about the relation between the total mass of the star and the core mass at which the helium flash takes place. The results by Rood (1972), Sweigart and Gross (1978), Lattanzio (1986) and Mazzitelli (1988) all agree within about $0.03 \mathrm{Me}$, and the agreement between the last two authors is even better than $0.015 \mathrm{Me}$. This small difference can perhaps be of some significance when studying the details in the HR diagram of the horizontal branch evolution (Caputo et Al 1984), but it is of no matter at all in the more general framework of the pre-white dwarf evolution. Also, agreement exists about the total and core mass for which a non degenerate helium core firstly ignites. Broadly speaking, a core mass about $0.48 \mathrm{M}$ can be assumed for degenerate ignition in the whole range 1.0 to $2.4 \mathrm{M}_{\theta}$ for a solar metal abundance and $\mathrm{x}=0.7$, and a core mass about $0.34 \mathrm{Me}$ for the star of $2.5 \mathrm{Mo}$ which ignites in nondegenerate conditions (Figure 1). Most unfortunately, if we allow for massive convective overshooting in main sequence (Roxburgh 1978, Maeder and Mermilliod 1981), the whole picture is dramatically modified. In fact, for those stars having a non negligible convective core in main sequence $(M>1.5 \mathrm{Mo})$, overshooting enlarges the core in such a way that, at the central hydrogen exaustion, a helium core about 0.33-0.35 $\mathrm{Me}$ is already present (Bertelli et Al 1986), which can gently ignite helium without degenerating. This leads to substantially lower core masses at helium ignition in the mass range $1.5-2.5 \mathrm{Me}$, and much larger core masses for $M>2.5 \mathrm{Me}$, since a general feature of the stellar evolution is that mass of the non degenerate helium core at the helium ignition increases almost linearly with the total mass of the star. Massive overshooting has been recently criticized, on apparently sound bases (Renzini 1987, Baker and Kuhfuss 1987); 
nevertheless, this possibility cannot at present be 'a priori' excluded, and I will briefly come back on this subject.

Going on to the central helium burning phase, up to the first thermal pulse, people working in stellar evolution well know the still open debate about the existence of large scale mixing mechanisms, such as semiconvection, overshooting on different scales, and breathing pulses or helium spikes (Robertson and Faulkner 1972, Sweigart and Renzini 1979, Castellani et al. 1985). All these mechanisms strongly affect the horizontal branch evolution, especially for Pop II stars. Luckily enough, it can be shown that, at least for Pop I stars in which a powerful hydrogen burning shell is present in any case, all the core mixing mechanisms have little influence upon the final hydrogen exausted core mass at the first thermal pulse.

Let me define $M_{H}$ the hydrogen exausted core mass, and $M_{\mathrm{H}} \mathrm{e}$ the helium exausted core mass. Without taking too seriously the following mathematics, we can write in a first approximation:

$$
\text { Ln LB }=C_{1} M_{B}+C_{2}
$$

which is a "Red Giant" relation between the shell hydrogen luminosity and the core mass. We can write also:

$$
\text { Ln Lre }=\mathrm{C}_{3} \mathrm{MH}_{\mathrm{H}}+\mathrm{C}_{4}
$$

which is instead a "helium main sequence" relation between helium luminosity and helium core mass. Moreover, we can obviously write:

$$
\mathrm{dM}_{\mathrm{H}} / \mathrm{dt}=\mathrm{C}_{\mathrm{s}} \mathrm{L}_{\mathrm{H}}
$$

$$
d M_{\text {E e }} / d t=C_{6} L_{H}
$$

where the various coefficients $C_{n}$ can be derived, partly from the models, partly from the first principles. Defining $t=0$ the beginning time of central helium burning, and $t$ ' the time of the first thermal pulse, from 4) and 2) one can write

5)

$$
M_{\text {Be }} \cdot=\int_{0}^{t^{\prime}} \exp \left(M_{H}\right) d t
$$

where $\mathrm{M}_{\mathrm{H}}$ ' is the helium exausted mass at the first thermal pulse. Moreover, from 3) and 1):

$$
\mathrm{dt}=\mathrm{dM} / \mathrm{C}_{8} \exp \left(\mathrm{M}_{\mathrm{B}}\right)
$$

and, substituting 6$)$ in 5) with the proper changing of the integration boundaries :

$$
M_{B} e^{\prime}=\int_{M_{1}}^{M_{M} e^{\prime}} C_{9} \exp \left(M_{B}\right) d M_{B}
$$



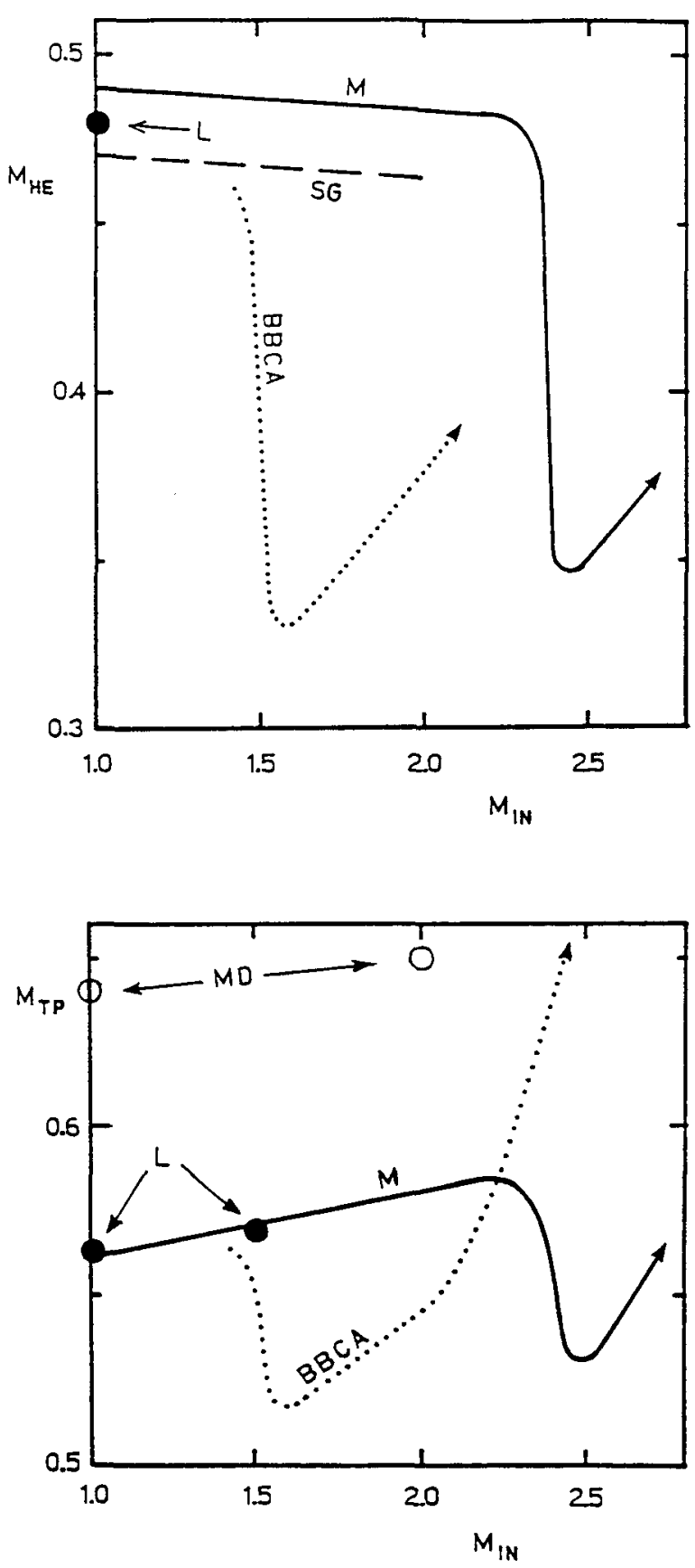

Figure 1: core mass at the central helium ignition for stars at helium flash. The continuous line (M) is relative to computations for the present paper; the point $L$ is from Lattanzio (1987): the dashed line (SG) is extrapolated from Sweigart and Gross (1978) and the dotted line (BBCA) is from Bertelli, Bressan, Chiosi and Angerer (1986), for models with extensive convective overshooting in main sequence.

Figure 2: Core mass at the first thermal pulse for the same range of initial masses as in Figure 1. The values labelied MD are from Mazzitelli and D'Antona (1986). 
being $M_{1}$ the hydrogen-exausted mass at the beginning of central helium burning, and the upper boundary having been put $M_{H} e^{\prime}$ instead of $M_{H}$ ' (the hydrogen exausted core mass at the first thermal pulse), since the two values are coincident within $1-2$ hundreths of solar mass. One has finally:

$$
C_{10} \exp \left(M_{\mathrm{B}} \mathrm{e}^{\prime}\right)-C_{11} \mathrm{M}_{\mathrm{B}} \mathrm{e}^{\prime}=C_{10} \exp \left(\mathrm{M}_{1}\right)
$$

that is: as long as 1) to 4) are verified, the final core mass at the first thermal pulse is a function of the initial core mass only, and not of the details of the helium-burning phase. In more plain words, as long as the Iuminosity of helium burning follows its main sequence like relation outlined in eq. 2), it is of no importanca at all where helium is burned (at the center, in a convective core or in a thick shell). The problem is rather to understand if eq 2) holds also in thick shell, and this seems to be the case according to the numerical models, at least for stars with masses not larger than $3 \mathrm{M}_{\theta}$, in which hydrogen and helium burning shells can coexist before the thermal pulses phase.

The behavior of the core mass at the first thermal pulse versus the total mass is shown in Figure 2 (Lattanzio 1986, 1987). As can be seen, almost all the stars undergoing the helium flash give rise to cores in the range $0.57-0.58$ Me unless, of course, mass loss during the first rise on the red giant branch has not already reduced the total mass of the star below these values. This is possible especially for stars of $1.0-1.2 \mathrm{Mo}$ with large mass loss rates, which can then die as low mass white dwarfs, possibly in the range $0.50-0.55 \mathrm{M}_{\theta}$.

Very different is the case if massive overshooting is present; definitely smaller core masses are found in the range $1.5-2.2 \mathrm{Mo}$. and much larger core masses for larger total masses (Bertelli et al. 1986). It is however to be recalled that these large differences are due to the differences in core mass at the beginning of the helium burning phase since, as shown before, the core mass at the first thermal pulse is quite insensitive to the behavior of central convection and, in any case, semiconvection and/or breathing pulses have the effect of mixing matter well beyond the formally convective core, almost in the same amount of large overshooting.

In Figure 2, also two values of core mass from Mazzitelii and D'Antona $1986 \mathrm{~b}$ are shown, and labeled as "upper limits". Care has to be taken with the chemical evolution scheme when performing this kind of computations, since the helium depletion during each time step is proportional to the third power of the helium abundance and, during the time step itself, the helium abundance decreases. In our 
computations of 1986, an algorithm was present which tended to overcorrect for this effect, giving rise to lower depletion rates and larger final core masses. In all the other computations, including the present ones and those by Lattanzio (1986, 1987), the abundance variation during the time step is instead computed according to the starting helium abundance, which causes an overestimate of the depletion and gives rise to lower core masses. It is to be expected that a better algorithm would lead to core masses somewhere in between the present values and those by Mazzitelli and D'Antona 1986.

Going ahead through the thermal pulse phase, it can be useful to note that the current calibration for the recurrency period of the thermal pulses versus the core mass (Paczynski 1975) probably gives smaller values than those presently found by several authors with updated input physics (Sweigart 1971, Gingold 1974, Iben 1975 and 1982, Schonberner 1979, Fujimoto 1979, Sackmann 1980, Wood and Zarro 1981, Lattanzio 1986, Mazzitelli 1987). Part of the difference is also due to the fact that Paczynski tuned the relation by computing a very few thermal pulses for each core mass, and all the computations show that at least for the first $10-12$ pulses, the interpulse period increases. It is then recommended to multiply by a factor $2-3$ the interpulse periods deduced by the Paczynski relation. This means, in turn, that the total number of thermal pulses experienced by a star during its asymptotic giant branch life is not very large; in the following we will see that it can be of the order of 30-40 in some cases but, for the more frequent cases, it can hardly exceed 10-20. Since the star at the first pulses is underluminous with respect to the equilibrium conditions, the following relations between asymptotic giant branch luminosity and core mass are suggested, holding also for the first pulses. Given a present core mass $M_{c}$, and a core mass $M^{\prime}$ at the first pulse, the steady interpulse luminosity and the peak surface luminosity at the pulse are respectively:

$\log L$ s $t e a d y / L \theta=3.15+1.33 M_{c}-0.3 \exp \left(M^{\prime}-M_{c}\right) / 0.015$

and

$\log L_{p e a k} / L_{\theta}=3.25+1.5 M_{c}-0.4 \exp \left(M^{\prime}-M_{c}\right) / 0.015$

The asymptotic branch phase is ultimately truncated by the loss of the hydrogen rich envelope by stellar wind or superwind (Renzini 1981). Most unfortunately, no complete theory based upon the first principles exists to be included in stellar evolution codes. For the wind, the empirical calibration by Reimers (1975) can be of some 
utility; for the superwind, only qualitative estimates of orders of magnitude, and indirect evidences exist. The main classes of mass loss mechanisms presently under investigation, upon which Linsky (1987) and Holzer (1987) have given extensive theoretical reviews, are:

-thermally driven winds, that is: steady state radial flow from the chromosphere. The gas density at the critical point (defined as the point where the outflow gas speed exceeds the sound speed) is however too low in all the cases to account for the observed mass loss rates. One has to invent mechanisms to increase the critical density, either by lifting matter in the corona, or shifting the critical point closer to the surface.

-Radiatively ariven winds, that is: radiation pressure on ions or, mainly, on circumstellar dust grains. Also in this case the mechanism seems not to work efficiently enough, since the stellar atmosphere theory predicts the grains to be very close to the surface, with greenhouse effect, melting of the grains, cooling, new grains formation and so on, but little or no wind.

-Periodic shock waves in pulsating stars, that is: periodic lifting of gas and grains due to pulsationally driven shock waves, with period shorter than the gravitational return time, so that in the end matter ejection occurs. This is probably the more clearly understood mechanism up today, but if it works for Mira variables, what about non-Miras?

-Alfven waves, that is: the same as before, but driven by hydromagnetic waves. Unfortunately, our present understanding of stellar magnetohydrodynamics is so poor that this mechanism is little more than a free parameter in the theory.

In summary, what we expect is heating and lifting of the atmosphere due to gravitational or hydromagnetic waves, so that the thermally and radiatively driven winds are largely powered. This gives no indication at all about the ignition of the superwind, so that the best the theory can say is that, if the maximum white dwarf mass which can be given birth from a given initial mass is computed according the Reimer's mass loss rate during the asymptotic giant branch evolution, and the minimum white dwarf mass is of course the core mass at the first thermal pulse, the superwind will give rise to a white dwarf mass somewhere in between these two limiting values. This situation is illustrated in Figure 3. The total number of thermal pulses ranges between 5 and 30 , and this is relevant when computing the expected enrichment in carbon and s-elements due to the third dredge-up.

As a result of the termination of the asymptotic giant branch phase during a continuous (wind or superwind) mass loss, the chances 
are that the blueward excursion for an object in this mass range begins during the interpulse phase, when a steady hydrogen burning shell is powering the star. In fact, from the computations it turns out that about $90 \%$ of the total mass lost from the star during a complete thermal pulse cycle is lost during the steady hydrogen burning phase. If it is so, the planetary nebulae nuclei should be hydrogen burners, according to schönberner (1987), taking also into account the complex picture outlined by Iben (1984) who follows also the case in which a final thermal pulse can take place during the blueward excursion, or the case in which, by chance, the blueward excursion itself begins during a thermal pulse. In practice, according to the above results, and considering that the initial mass function for main sequence stars is peaked around the lower limits, it is to be expected that more than $80 \%$ of the white dwarfs have their origin in this way, and more than $70 \%$ of them die with a still thick hydrogen envelope, apart of course from the possible depletion of surface hydrogen due to wind in the luminous blue region of the HR diagram.

\section{STARS GENTLY IGNITING HELIUM}

Let me now go ahead to the second mass range that is: stars gently igniting helium at the center, and not undergoing the second dredge-up. For these stars, the relation between core mass at the helium ignition and initial mass is not flat any more as it was for the first mass range, but the core mass steeply increases, almost linearly with total mass. Of course, also the envelope mass linearly increases and, in principle, this would mean that such stars should experience a larger and larger number of thermal pulses before wind or superwind can stop the asymptotic giant branch evolution. Actually, not only the observations (Iben 1981), but also some theoretical considerations suggest that the game is played in a different way. In fact, when the core mass increases due to the ongoing thermal pulses, both the steady interpulse and peak-of-the-pulse surface luminosities increase, the latter with a $3 / 2$ power of the core mass. At a given point we see from the models that, at the peak of the surface luminosity during a thermal pulse, when the hydrogen shell is completely turned off, several processes occur in the envelope, mainly:

the hydrogen rich envelope is cooled, lifted and expanded;

-the temperature at the base of the convective envelope drops to a few in $10^{5} \mathrm{~K}$, and Hydrogen recombines in almost the whole envelope;

-the total energy of the envelope (including the dissociation and ionization energy) becomes larger than the gravitational binding 
energy lof course this does not necessarily mean that the ejection of the envelope is unavoidable, since one has to estimate the conversion efficiency into kinetic energy);

-the radiation pressure at the $\mathrm{H}-\mathrm{He}$ interface dominates by orders of magnitude (it can be easily more than $99 \%$ of the total pressure);

-in the helium rich layers just behind the base of the convective envelope, the mean free path of a photon begins to be a non negligible fraction (some hundreths) of the local stellar radius, so that the radiation field is not isotropic any more, but there is a net radiation flux toward the external layers.

In these conditions, not only the evolutionary computations begin to become very hard, since the specific heat of matter is higly non linear with respect to the thermodynamical quantities, but also the approximation of local isotropy of the energy flux is no longer valid and the numerical results obtained in this hypotesis can be no longer reliable.

In practice, several authors, working in different structural or evolutionary frameworks, have found that a sudden loss of the hydrogen rich envelope it is to be expected at the peak of a thermal pulse, when the core mass ranges between 0.8 and $0.9 \mathrm{Me}$. I can quote the hydrodynamic computations by Kutter and sparks (1974) for a structure with a helium core powering the luminosity and a turned off hydrogen envelope, the results by Tuchman et Al. (1978) and Barkat and Tuchman (1979) showing that the envelope of a Mira variable at the maximum is unbound and can be ejected beyond a given luminosity, the difficulties met by Faulkner and Wood (1984) in computing advanced thermal pulses, which lead the same wood and Faulkner (1986) to hypotize the reaching of an "Eddington - like" luminosity at the base of the envelope, and a similar result by myself (Mazzitelli 1987) in the computation of a long run of thermal pulses, with the ratio between mean free path of photons and local radius exponentially increasing from the peak of one pulse to the other.

As can be seen, the effects quoted above are completely different from each other, and seem to have nothing in common; actually, at the very base of all of them there is the enormous lifting and cooling of the hydrogen rich envelope, which causes recombination, large increase in opacity, and the storage of a large amount of energy in the envelope itself. In spite of the uncertainties in the theory, we can reasonably safely conclude that, in these conditions, a detachment of the hydrogen rich envelope is very likely at the maximum surface luminosity at the peak of a thermal pulse, when the core mass is about $0.85 \mathrm{M}$ (depending on the total mass) and the steady interpulse 

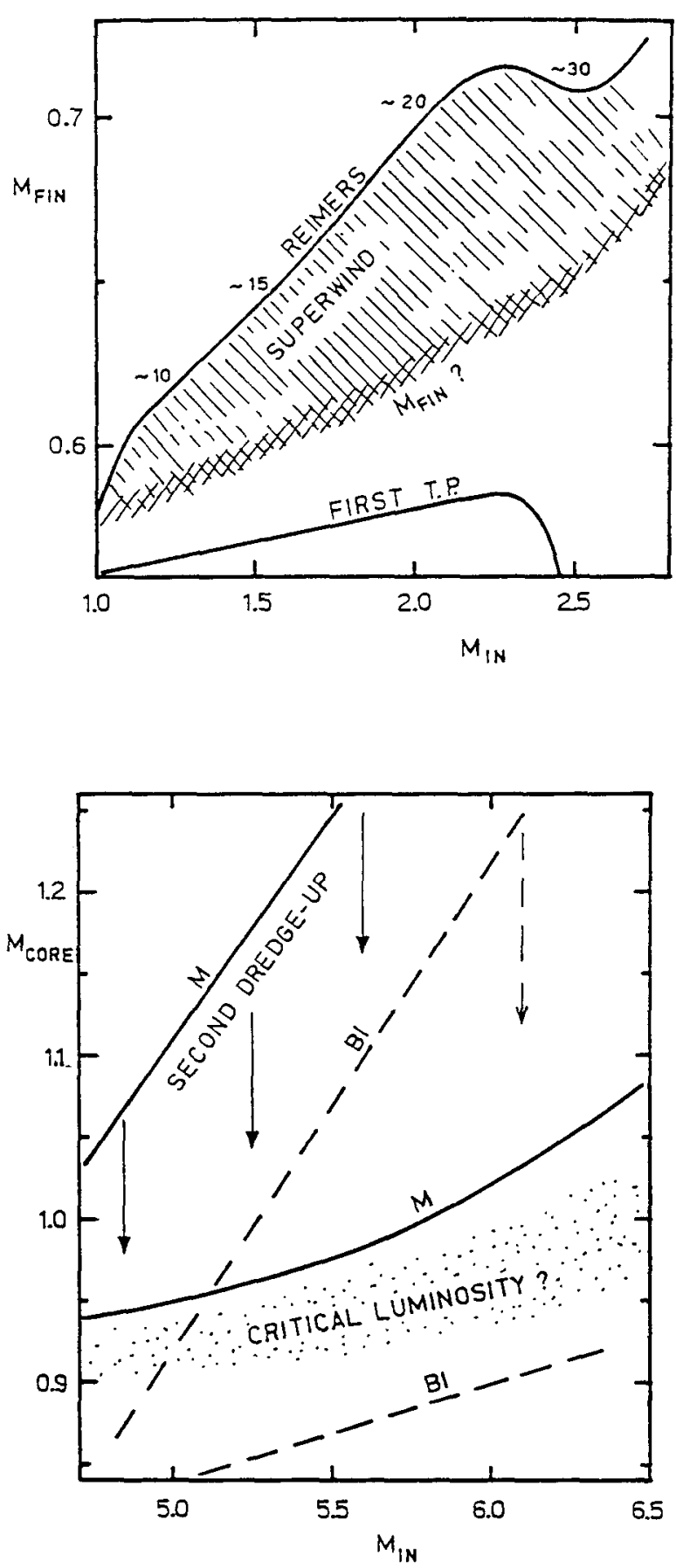

Figure 3: the relation between $M_{1 n}$ and $M_{f 1 n}$ according to the normal wind (Reimers) and the superwind. The approximate numbers of thermal pulses experienced by stars of various masses are also shown.
Figure 4: the core mass before and after the second dredge-up are shown for the computations by Becker and Iben (1979, BI dashed lines), and for the present computations ( $M$ continuous lines). Also the expected region where the envelope is ejected for critical luminosity is shown. 
luminosity is around Log $\mathrm{L} / \mathrm{L}_{\theta}=4.3$, and this is the mechanism terminating the asymptotic giant branch phase for these stars. Note that, if this is the real occurrence, we can expect very thin hydrogen envelopes upon the white dwarfs originated in this way: in fact, if the hydrogen opacity is the triggering mechanism for the envelope ejection, almost no hydrogen (probably enough for an optical atmosphere, but not for hydrogen burning in the blue) should be left at the surface of the star. We further expect a steep slope in the $M_{f} 1 \mathrm{n}-M_{1 n}$ relation for masses in the range $2.5-4 M_{\theta}$, since also the mass at the beginning of the helium burning phase sharply increases with total mass. For $M_{1}>4 M_{\theta}$ we then expect a much flatter behaviour, since, for these stars, the evolution in asymptotic giant branch terminates for core masses in a relatively narrow range 10.8 to 0.9 Mo). Also note that the total number of thermal pulses experienced by these stars reaches a maximum of the order of 50 around $3-3.5 \mathrm{Mo}$, then fastly decreases to a very few or no thermal pulses at all for larger masses.

\section{STARS EXPERIENCING THE SECOND DREDGE-UP}

I will finally discuss the last mass range, that is stars experiencing the second dredge-up (Becker and Iben 1979). The core masses before and after the dredge-up as a function of the total mass are shown in Figure 4 , where both the results by Becker and Iben and a set of results computed by myself for the present talk are collected. There are some differences due, only in part, to updating of the input physics. One major difference, which unfortunately throws a shade upon the reliability of all these results, is that at the maximum deepening of surface convection during the second dredge-up, all the convective region, down to the base just above the helium burning shell, is largely overadiabatic. This could not be found with previous computer codes, in which the overadiabaticity is considered only in the external subatmospheric layers, but I have found it in the present computations since, being the code a full Raphson-Newton up to the base of the optical atmosphere, overadiabatic convection had to be included all through the structure (in these computations, the ratio of mixing length to pressure scale height is 1.0). As an example, for the star of $6.5 \mathrm{Mo}$, an average overadiabatic gradient about 0.2 was found down to the bottom of the convective envelope, around $1.1 \mathrm{M}_{\theta}$. When recalling that these envelopes are highly dominated by radiation pressure, so that the adiabatic gradient is very close to 0.25 , the 
consequences of such large overadiabaticity can be easily understood, at least from the side of the reliability of the models. Lacking any better physical treatment than the mixing length theory, we are forced to assume that the stellar models be reliable at least as long as overadiabatic convection is present in thin subatmospheric layers only; it is however hard to believe that a theory which can be responsible for large scale density inversions along more than $80 \%$ of a stellar structure can provide physically sound models, to be compared to the observations. In my opinion this is presently another of the boundaries within which the theory of stellar evolution has to be forced.

In the hope that all the models dealing with this phase are not completely meaningless, let me go on to the discussion of the evolution to white dwarf for this mass range. Actually, the general structure of the star at the maximum deepening of the second dredge-up closely resembles the structure at the peak of a thermal pulse. Also in this case the hydrogen rich envelope is cold and enormously expanded (the density at the base of the envelope can be $10^{-6}$ or $10^{-7}$ $\mathrm{g} / \mathrm{cc}$, and so on, so that it is likely that the envelope itself be blown up without even igniting the first thermal pulse or, since the helium remnant layer above the helium burning shell is, at the maximum penetration of surface convection, still of several hundreths of $\mathrm{Me}_{\mathrm{e}}$, very few thermal pulses can occurr, due to thermal adjustment of the helium layer. The possibility that stars in this mass range die without previously going through a long run of thermal pulses has already been indicated for instance by Iben (1987), on the ground that asymptotic giant branch stars of this kind are by far undernumerous.

Unfortunately, if this is true, we are left with a severe problem. In fact, white dwarfs with masses larger than $1.05-1.1 \mathrm{M}$ were thought to be originated by asymptotic giant branch stars with initial core masses smaller than these values, but undergoing long runs of thermal pulses, and accreting mass upon the core up to the theoretical Chandrasekhar limit since, as already quoted at the beginning, a carbon - oxygen core born with a mass larger than 1.05 $1.1 \mathrm{Me}$ (depending on the carbon abundance) very fastly ignites. In the above outlined framework, there is no room for white dwarfs of mass larger than $1.05-1.1 \mathrm{Me}$, unless they are formed after a violent but not catastrophyc phase of carbon burning, following degenerate ignition, and without the destruction of the structure. In this case, large mass white dwarfs should be formed by $22 \mathrm{Mg}$ and $24 \mathrm{Mg}$, but no theoretical models showing this possibility are presently available so that the existence of these white dwarfs is still not explained. 


\section{CONCLUSIONS}

In summary, the relation $M_{f 1 n}-M_{1}$ for the whole range of initial masses of interest is summarized in Figure 5, together with a recent semiempirical relation suggested by Weidemann (1987a). The agreement for low initial masses is quite good, but it is an artificial byproduct of the fact that, knowing almost nothing about the theoretical superwinds, I simply draw a bona fide band not far from the semiempirical calibration. For larger masses, there is a sistematic difference about $0.15 \mathrm{Mo}$ with respect to the calibration by Weidemann, but I do not think this disagreement be dramatic since, up today, due to the low statistics, the semiempirical relation is not easily tuned in this region. It is interesting to note that, if the relation follows indeed the band drawn in Figure 5, the mass distribution function of the observed white dwarfs has to show a peculiar behavior. After a peak around $0.6 \mathrm{Mo}$ and a sharp decrease up to $0.85 \mathrm{Me}$, the mass function shows a flattening, if not a secondary (very low) peak around $0.9 \mathrm{Mo}$, to drop again for larger masses.

Actually, this seems to be the case (Weidemann 1987b), although the statistics is too low to be reliable, and we will have to wait perhaps the Hubble space Telescope for an answer. This theoretical prediction, which could be probably confirmed or ruled out by the observations in a few years, can be a reasonable conclusion for the review.

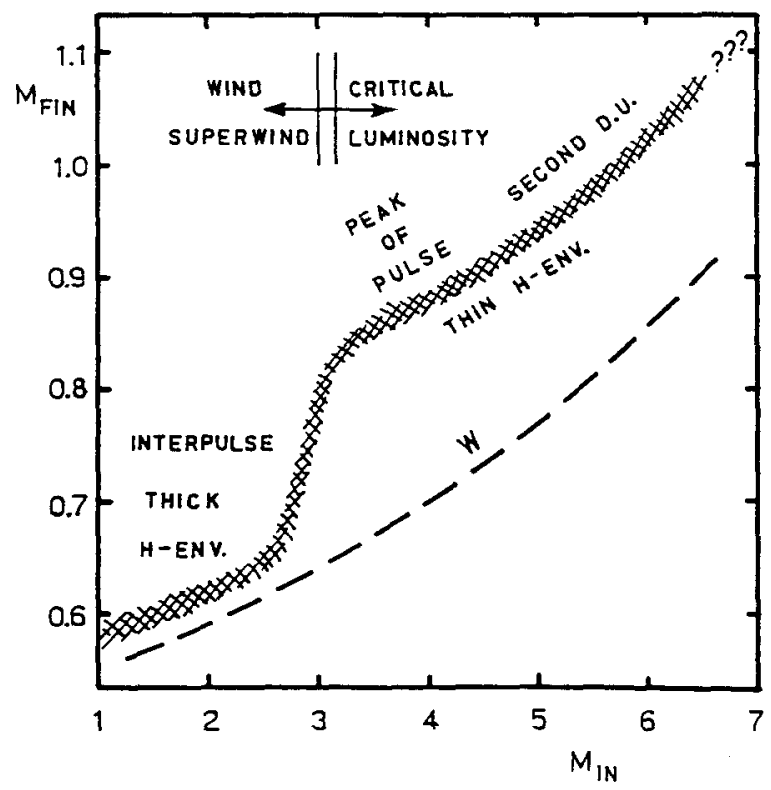

Figure 5: Mfin vs. Min relation for the range $1-7$ Mo, together with the semiempirical relation (dashed line) obtained by Weidemann (1987a). 
Baker, N. H. , Ruhfuss, R. 1987, Astron. Astrophys. 185, 117.

Barkat, Z.. Tuchman, Y. 1979, Astrophys. J. 237, 105.

Becker, S. A., Iben, I. Jr., 1979 Astrophys. J. 232, 831.

Bertelii, G., Bressan, A., Chiosi, C., Angerer, R. 1986, Astron. Astrophys. Suppl. 66, 191.

Caputo, F., Castellani, V., Di Gregorio, R., Tornambe', A. 1984 Astron. Astrophys. Suppl. 55, 463.

Castellani, V.. Chieffi, A., Pulone, L., Tornambe', A., 1985a, Astrophys. J. Lett. 294, L31.

Castellani, V., Chieffi, A., Pulone, L., Tornambe', A., 1985b, Astrophys . J. 296, 204.

$D$ Antona, F., 1988, in this volume.

Faulkner, D.J., Wood, P.R. 1984, Proc. Astr. Soc. Australia, 5, 543.

Fujimoto, M. Y., 1979 Publ, Astron. Soc. Japan 31, 1.

Gingold, R. A. , 1974 Astrophys. J. 193, 177.

Holzer, T.E. 1987, in IAU symposium No. 122, Circumstellar Matter, ed. I. Appenzeller and C. Jordan (D. Reidel Publ. Co.), 289.

Iben, I. Jr., 1975 Astrophys. J. 196, 525.

Iben, I. Jr., 1981, Astrophys. J. $\frac{246}{24}, 278$

Iben, I. Jr., 1982 Astrophys. J. 253, 248.

Iben, I. Jr., Raler, J. B., Truran, J., W. and Renzini, A. 1983. Astrophys. J. 264, 605.

Iben, I. Jr. 1984, Astrophys. J. 277, 333.

Iben, I.Jr., Tutukov, A. V. 1984, Astrophys. J. 282, 615.

Iben, I. Jr. 1987, in Late stages of stellar Evolution, S. Kwok and S. R. Pottasch ed. (Reidel, Dordrecht), p. 175.

Rutter, G. S., Sparka, W. M. 1974, Astrophys. J. 192, 447.

Lattanzio, J . C. 1986, Astrophys. J. 311, 708 .

Lattanzio, J.C. 1987, in Late stages of stellar Evolution, S. Kwok and S. R. Pottasch ed., (Reidel,Dordrecht), p. 235.

Linsky, J. L. 1987, in IAU symposium No. 122, Circumstellar Matter, ed. I. Appenzeller and C. Jordan (D. Reidel Publ. Co.), 271.

Maeder, A.. Mermilliod, J. C. 1981, Astron. Astrophys. 93, 136.

Mazzitelii, I., D'Antona, F. 1986a, Astrophys. J. 308, 706.

Mazzitelli, I., D'Antona, F. 1986b, Astrophys. J. 311, 762.

Mazzitelli, I., D'Antona, F. 1987, in IAU Colloguium No. 95 The Second Conference on Faint Blue Stars, L.Davis Press p. 351.

Mazzitelli, I. 1987, in 6 th European Workshop on White Dwarfs, Mem. Soc. Astron. Ital. 58, 117 .

Mazzitelii, I. 1988, Astrophys, J. in press.

Paczynski, B. 1975, Astrophys. J. 202, 558 .

Reimers, D. 1975, Mem. Soc. Roy. Sci. Liege, Ge Ser., $8,369$.

Renzini, A. 1981, in Physical Processes in Red Giants, I. Iben Jr. an A. Renzini Eds... Reidel, Dordrecht, p. 431.

Renzini, A. 1987, Astron. Astrophys. $188,49$.

Robertson, J. W.. Faulkner D. J. 1972, Astrophys. J. 171, 309.

Rood, R. T. 1972, Astrophys. J. 177,681 .

Roxburgh, I. 1978, Astron. Astrophys. $\underline{65}, 281$.

Seckmann, I. J. 1980 , Astrophys. J. 235,960 .

Schonberner, D. 1979, Astron. Astrophys. $79,108$.

Schbnberner, D. 1981, Astron. Astrophys. $103,119$.

Schonberner, D. 1983, Astrophys. J. 272, 708 .

schönberner, D. $1987 \mathrm{a}$, in IAU Colloquium No. 95 The Second Conference On Faint Blue Stars, L.Davis Press p. 201.

Schónberner, D. $1987 \mathrm{~b}$, in Late stages of steliar Evolution,

S. Kwok and S. R. Pottasch ed., p. 337.

Sweigart, A. V. 1971, Astrophys. J. 168, 79.

Sweigart, A. V., Gross, P. G. 1978, Astrophys. J. Supp1. 36, 405.

Sweigart, A. V., Renzini, A. 1979, Astron. Astrophys. 71, 66 .

Tuchman, Y., Sach, N., Barkat, Z. 1978, Astrophys. J. $219,183$.

Weidemann, v. 1987a, in Late stages of stellar Evolution,

S. Kwok and S. R. Pottasch ed., D. 347 .

Weidemann. V. $1987 \mathrm{~b}$, in IAU Colloquium No. 95 The Second Conference on Faint Blue Stars, L.Davis Press p. 19.

Wood, P. R., Faulkner, D. J. 1986, Astrophys. J. 307, 659.

Wood, P. R., Zarro, D. M. 1981, Astrophys. J. 247, 247. 